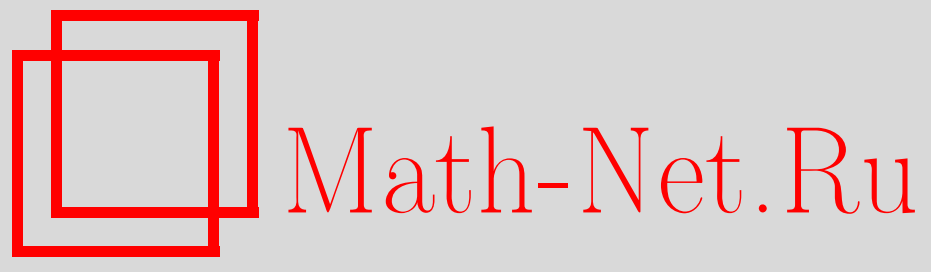

И. С. Ярославцев, Об асимметрии прошлого и будущего эргодического $\mathbb{Z}$-действия, Матем. заметки, 2014, том 95, выпуск 3, 479-480

DOI: https://doi.org/10.4213/mzm10448

Использование Общероссийского математического портала Math-Net.Ru подразумевает, что вы прочитали и согласны с пользовательским соглашением http://www . mathnet.ru/rus/agreement

Параметры загрузки:

IP: 54.237 .206 .68

26 апреля 2023 г., 11:28:46






\section{Об асимметрии прошлого и будущего эргодического $\mathbb{Z}$-действия}

\section{И. С. Ярославцев}

Задача неизоморфизма (асимметрии) сохраняющего меру преобразования и его обратного имеет давнюю историю в эргодической теории (см, например, [1]). В работе [2] в качестве инварианта, различающего действие и его обратное, используется кратная возвращаемость. Этот подход был развит и нашел применения в [3]-[5].

Цель заметки - упростить конструкцию преобразования и доказательство его асимметрии, следуя методу [1]. Мы получим существенно разные пределы выражений

$$
\mu\left(F \cap T^{n_{j}+1} G \cap T^{2 n_{j}} H\right) \quad \text { и } \quad \mu\left(F \cap T^{-n_{j}-1} G \cap T^{-2 n_{j}} H\right) .
$$

Один из них будет иметь составляющую $\mu(F \cap G \cap H) / 3$, а второй - нет. Этот инвариант (относительно сопряжения) и будет различать $T$ и $T^{-1}$.

Теорема. Существует эргодическое преобразование T, обладающее следующим свойством: для некоторой последовательности $n_{j} \rightarrow \infty$ для любых измеримых множеств $F$, $G$ и Н выполнено равенство

$$
\begin{aligned}
\lim _{j \rightarrow \infty} & \mu\left(F \cap T^{n_{j}+1} G \cap T^{2 n_{j}} H\right) \\
& =\frac{1}{3}\left(\mu\left(F \cap T G \cap T^{-1} H\right)+\mu\left(F \cap T^{2} G \cap T H\right)+\mu(F \cap G \cap H)\right) .
\end{aligned}
$$

СлЕДСтвиЕ. Преобразования $Т$ и $T^{-1}$ неизоморфны.

ДокАзАтЕльство. Из теоремы непосредственно вытекает

$$
\begin{aligned}
\lim _{j \rightarrow \infty} & \mu\left(H \cap T^{-n_{j}-1} G \cap T^{-2 n_{j}} F\right)=\lim _{j \rightarrow \infty} \mu\left(F \cap T^{n_{j}-1} G \cap T^{2 n_{j}} H\right) \\
= & \frac{1}{3}\left(\mu\left(F \cap T^{-1} G \cap T^{-1} H\right)+\mu(F \cap G \cap T H)+\mu\left(F \cap T^{-2} G \cap H\right)\right) .
\end{aligned}
$$

Пусть множество $A$ положительной меры таково, что $A, T A$ и $T^{2} A$ попарно не пересекаются. Тогда

$$
\lim _{j \rightarrow \infty} \mu\left(A \cap T^{n_{j}-1} A \cap T^{2 n_{j}} A\right)=0 .
$$

Предположим, что существует сохраняющее меру преобразование $R$ такое, что $T^{-1}=$ $R^{-1} T R$. Покажем, что

$$
\lim _{j \rightarrow \infty} \mu\left(A \cap T^{n_{j}-1} A \cap T^{2 n_{j}} A\right) \geqslant \frac{1}{3} \mu(A),
$$

тем самым получим противоречие с предыдущим утверждением. Действительно,

$$
\begin{aligned}
\lim _{j \rightarrow \infty} & \mu\left(A \cap T^{n_{j}-1} A \cap T^{2 n_{j}} A\right)=\lim _{j \rightarrow \infty} \mu\left(T^{-2 n_{j}} A \cap T^{-n_{j}-1} A \cap A\right) \\
& =\lim _{j \rightarrow \infty} \mu\left(A \cap R^{-1} T^{n_{j}+1} R A \cap R^{-1} T^{2 n_{j}} R A\right)=\lim _{j \rightarrow \infty} \mu\left(R A \cap T^{n_{j}+1} R A \cap T^{2 n_{j}} R A\right) \\
& \geqslant \frac{1}{3} \mu(R A)=\frac{1}{3} \mu(A) .
\end{aligned}
$$

ДОКАЗАТЕЛЬСТВо тЕОРЕМЫ. По аналогии с конструкцией в работе [2] найдется преобразование $T$, последовательность $h_{j} \rightarrow \infty$ и последовательность разбиений фазового пространства

$$
X=Y_{j} \sqcup Y_{j}^{1} \sqcup Y_{j}^{2} \sqcup Y_{j}^{3}
$$

DOI: $10.4213 / \mathrm{mzm} 10448$ 
такие, что $\mu\left(Y_{j}\right) \rightarrow 0$, а множества $Y_{j}^{1}, Y_{j}^{2}, Y_{j}^{3}$ имеют следующий вид:

$$
\begin{aligned}
Y_{j}^{1} & =B_{j}^{1} \cup T B_{j}^{1} \cup \cdots \cup T^{h_{j}-2} B_{j}^{1} \cup T^{h_{j}-1} B_{j}^{1}, \\
Y_{j}^{2} & =B_{j}^{2} \cup T B_{j}^{2} \cup \cdots \cup T^{h_{j}} B_{j}^{2} \cup T^{h_{j}+1} B_{j}^{2}, \\
Y_{j}^{3} & =B_{j}^{3} \cup T B_{j}^{3} \cup \cdots \cup T^{h_{j}-1} B_{j}^{3} \cup T^{h_{j}} B_{j}^{3} .
\end{aligned}
$$

Потребуем также, чтобы выполнялось

$$
\begin{gathered}
T^{h_{j}} B_{j}^{1}=B_{j}^{2}, \quad T^{h_{j}+2} B_{j}^{2}=B_{j}^{3}, \quad \frac{\mu\left(T^{h_{j}+1} B_{j}^{3} \Delta B_{j}^{1}\right)}{\mu\left(B_{j}^{1}\right)} \rightarrow 0, \\
\xi_{j}=\left\{C_{j}, T C_{j}^{1}, T^{2} C_{j}, \ldots, T^{h_{j}-1} C_{j}\right\} \rightarrow \varepsilon,
\end{gathered}
$$

где $C_{j}=B_{j}^{1} \cup B_{j}^{2} \cup B_{j}^{3}$, а запись $\xi_{j} \rightarrow \varepsilon$ означает, что любое измеримое $A$ аппроксимируется $\xi_{j}$-измеримыми множествами.

Положим $n_{j}=h_{j}+1$. Для любых измеримых $F, G, H$ выполнено

$$
\begin{aligned}
\mu\left(Y_{j}^{1} \cap F \cap T^{n_{j}+1} G \cap T^{2 n_{j}} H\right) & \rightarrow \frac{1}{3} \mu\left(F \cap T G \cap T^{-1} H\right), \\
\mu\left(Y_{j}^{2} \cap F \cap T^{n_{j}+1} G \cap T^{2 n_{j}} H\right) & \rightarrow \frac{1}{3} \mu\left(F \cap T^{2} G \cap T H\right), \\
\mu\left(Y_{j}^{3} \cap F \cap T^{n_{j}+1} G \cap T^{2 n_{j}} H\right) & \rightarrow \frac{1}{3} \mu(F \cap G \cap H) .
\end{aligned}
$$

Проверка этих утверждений стандартна: вместо множеств $F, G, H$ следует на каждом $j$-м шаге подставлять $\xi_{j}$-измеримые множества, которые аппроксимируют $F, G, H$, а затем перейти к пределу. Сложив последние три передела и учитывая, что

$$
\mu\left(X /\left(Y_{j}^{1} \sqcup Y_{j}^{2} \sqcup Y_{j}^{3}\right)\right) \rightarrow 0,
$$

получим утверждение теоремы.

Автор признателен В.В. Рыжикову за консультации и следующее замечание. Рассмотренное свойство асимметрии кратного возвращения является типичным свойством: множество таких преобразований массивно относительно естественной метрики на группе всех сохраняющих меру преобразований. С. В. Тихонов, благодаря введению новой метрики [6], развил теорию типичных перемешивающих преобразований (см. также [7], [8]). В [6] им была доказана типичность асимметрии. Хотя используемый нами метод не может быть применен к перемешивающим системам, он работает для частично перемешивающих систем, сколь угодно близких к перемешивающим.

\section{СПИСОК ЦИТИРОВАННОЙ ЛИТЕРАТУРЫ}

[1] В.И. Оселедец, Функи. анализ и его прил., 5:3 (1971), 75-79. [2] В. В. Рыжиков, Динамические системь и смежные вопросы, Тр. МИАН, 216, Наука, М., 1997, 154-157. [3] В. В. Рыжиков, Матем. заметки, 74:6 (2003), 889-895. [4] A. I. Danilenko, V. V. Ryzhikov, Proc. Lond. Math. Soc. (3), 104:3 (2012), 431-454. [5] K. Fraczek, J. Kulaga, M. Lemanczyk, "Non-reversibility and self-joinings of higher orders for ergodic flows", J. Anal. Math. (to appear); arXiv: math.DS/1206.3053. [6] С. В. Тихонов, Матем. сб., 198:4 (2007), 135-158. [7] С. В. Тихонов, Матем. заметки, 90:6 (2011), 953-954. [8] А. И. Баштанов, Матем. заметки, 93:2 (2013), 163-171.

И. С. Ярославцев

Поступило

Московский государственный

11.12 .2013

университет им. М. В. Ломоносова

E-mail: Yaroslavtsev.I.S@yandex.ru 